\title{
47. Disfacilitation as the Basis for the Sensory Suppression in a Specialized Lateralis Receptor of the Marine Catfish
}

\author{
By Shosaku OBARA*) and Yutaka OomurA \\ Department of Physiology, Kanazawa Univ., Faculty of Med., Kanazawa 920
}

(Comm. by Yasuji Katsuki, M. J. A., March 12, 1973)

The directional sensitivity of the acoustico-lateralis receptor has been attributed to the polarized position of the kinocilium at the lumenal face of the receptor cell. ${ }^{2)}$ The microphonics analysis has been also correlated with the afferent nerve activity. ${ }^{2), 5)}$ Namely, i) the receptor cell is depolarized or hyperpolarized by mechanical stimuli in opposite directions with respect to the kinocilium, ii) the receptor response exhibits a marked non-linearity, the depolarization being much larger in size than the hyperpolarization and iii) the receptor de- and hyperpolarization lead synaptically to a facilitation and a suppression of the afferent discharge. Detailed mechanisms for these steps, however, remain largely unknown. The receptor de- and hyperpolarization have been reported to result from vastly different ionic mechanisms in various receptor systems. ${ }^{3)}$ Although an EPSP has been shown in the eighth nerve of goldfish, ${ }^{5)}$ the receptor-afferent transmission of the acoustico-lateralis system in general is still subject to several theoretical possibilities. ${ }^{3)}$ The present report will describe the disfacilitation in a specialized lateralis receptor, which implies that the receptor-afferent transmission is mediated by a single excitatory transmitter.

The tonic electroreceptors, the specialized lateralis receptor, present an interesting parallelism to the ordinary lateralis receptor, both in their synaptic organization ${ }^{1), 8}$ and in their mode of operation, except that the increase or decrease of the afferent output is determined by the polarity of the effective voltage gradient across the sensory epithelium. ${ }^{1), 6), 9), 10)}$ Of particular interest are the ampullae of Lorenzini, in that their unique structure allows one to readily expose the ampullae (receptive elements) and the afferent nerve terminals for microelectrode survey under direct visual observation.9),10) The experiments were carried out in situ on the ampullae of Lorenzini of the marine catfish, Plotosus anguillaris (Lacépède). The fish was curarized and maintained by perfusing sea water through the gill, and the preparation remained in a steady condition for several hours, unless

*) Present address: Department of Physiology, Teikyo Univ., School of Med., Kaga 2-11-1, Itabashi-ku, Tokyo 173 
A

B

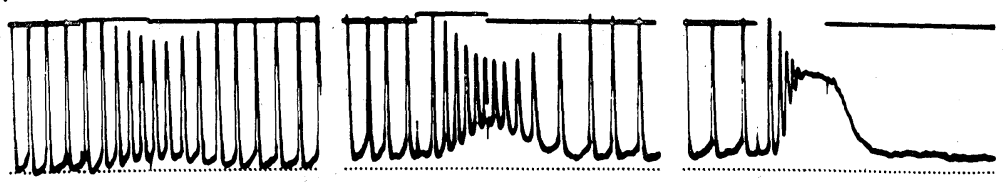

D

E
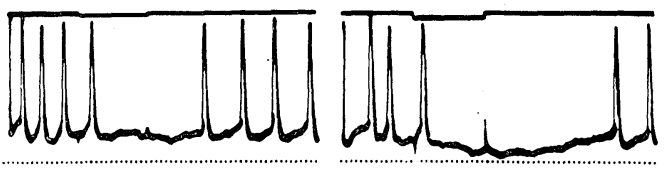

$F$
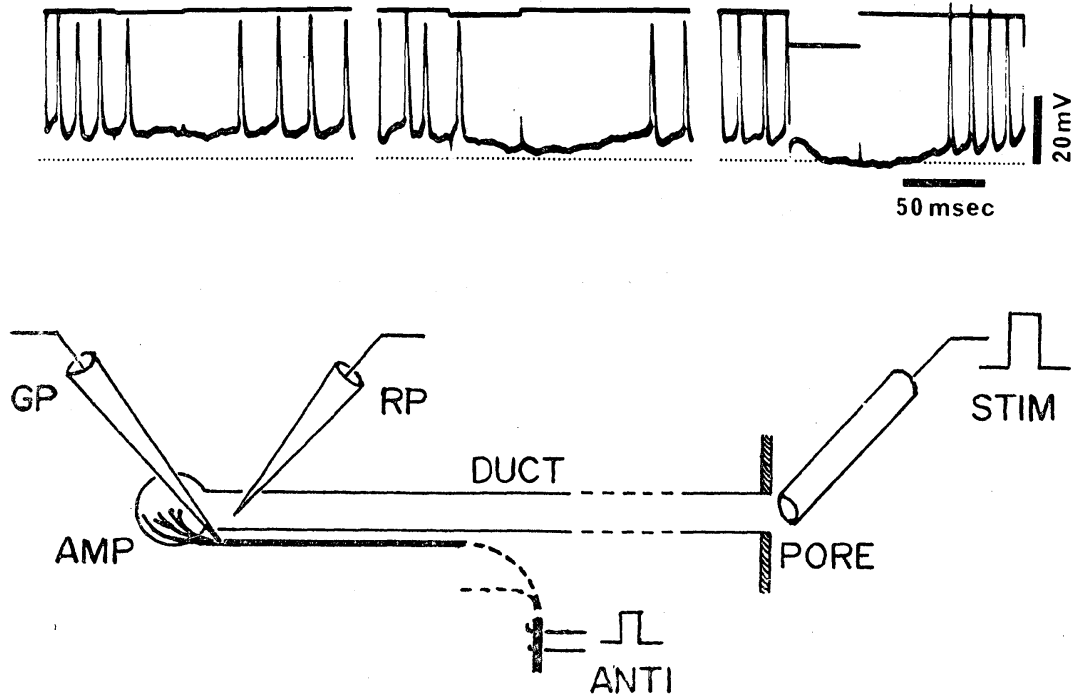

Fig. 1. Responses in the afferent terminal to the electrical stimulation to the pore. Upper trace: the stimulus (the pore opens in sea water, so that the stimulus signal is proportional to, but much larger than the effective stimulus to the ampulla). Lower trace: the intra-axonal recording. Stimulation with anode at the pore in $\mathrm{A}$ to $\mathrm{C}$, and that with cathode in $\mathrm{D}$ to $\mathrm{F}$. The dotted lines indicate the lowest level of the axonal membrane potential. Bottom figure: Diagram of the arrangement for recording and stimulation. AMP: the ampulla, with its afferent innervation shown as a thick line. DUCT: the long radiating duct from individual ampulla which opens to exterior at distant PORE. RP and GP: recording electrodes in the ampullary lumen and in the afferent terminal. STIM: stimulation to the respective pore. ANTI: antidromic stimulation to the nerve trunk.

the fine blood capillary network to the ampullae was disturbed. Responses of the receptor ( $\mathrm{RP}$, receptor potential) were recorded in the ampullary lumen by a glass microelectrode, and taken as a mass response of many receptor cells aligned in parallel in the ampulla wall.4),8,10) Intra-axonal recording from the afferent terminal revealed graded slow potential changes in either direction on stimulation together with the spike discharge (Fig. 1). Since the slow potential in depolarizing direction is associated with the facilitation of the afferent discharge ( $\mathrm{A}$ to $\mathrm{C}$ ) and the hyperpolarization with the suppression of 

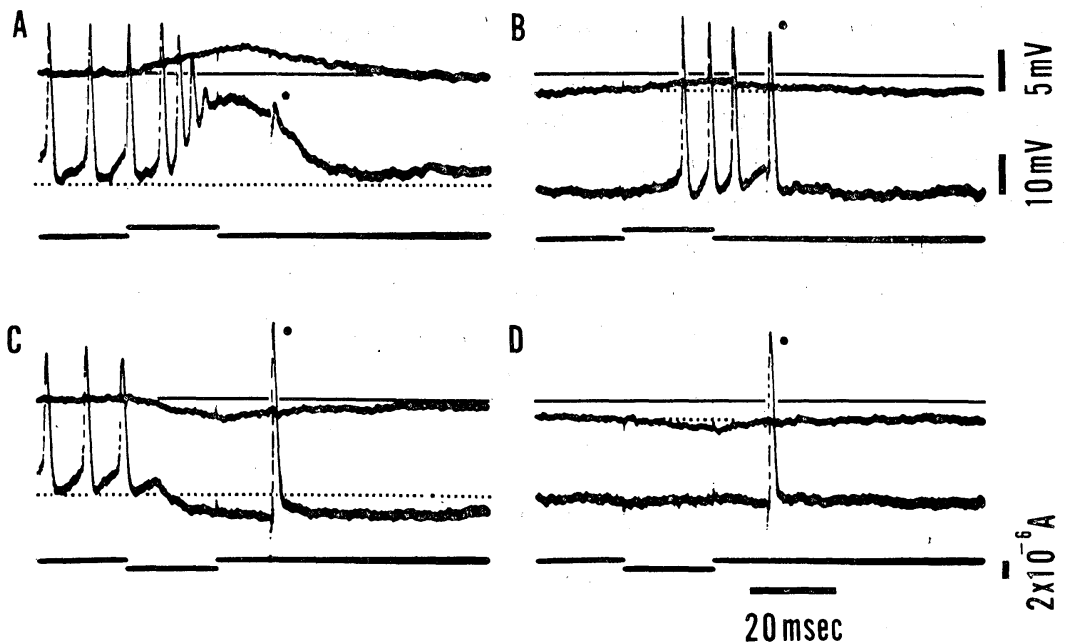

Fig. 2. Simultaneous recording of the ampullary and intra-axonal potentials. Uppermost trace: the potential in the ampullary lumen, with the straight line drawn at the control level. Additional dotted lines were placed at the prestimulus level in $B$ and $D$ which indicate the lowest level of the ampulla potential. Middle trace: the intra-axonal potential. The antidromic spikes are marked by dots near their peak. The dotted lines in $\mathrm{A}$ and $\mathrm{C}$ were drawn at the lowest level of the axonal potential during the resting discharge. Lowermost tracia the stimulus as in Fig. 1. Note the decrease of the antidromic spike height during the depolarizing GP (A and B), and the increase of which during the hyperpolarizing GP (C). For further explanation see text.

the latter ( $\mathrm{D}$ to $\mathrm{F}$ ), they will be called the depolarizing and hyperpolarizing GP, generator potential (or PSP), respectively. ${ }^{9), 10)}$

As shown in Fig. 2A, an anodal current pulse given at the pore produced a positive-going RP in the ampulla, which clearly outlasted the stimulus. Concomitant with the RP, a large depolarizing GP was observed in the afferent terminal. Since the sensitive system (with threshold in the order of 1 to 10 microvolts ${ }^{10}$ ) was overdriven beyond its linear working range, the excessive depolarizing GP rapidly inactivated the afferent spike generation (Fig. 2A, and also Fig. 1C). Applying the same stimulus during a suppressed period (Fig. 2B), a near maximum facilitation of the afferent discharge from complete shut-off was produced by a small RP in the ampulla (less than $0.8 \mathrm{mV}$ in this case). A cathodal pulse of the same intensity evoked a negative-going RP in the ampulla, and a prolonged hyperpolarizing GP in the afferent terminal with corresponding suppression of the afferent discharge. (Fig. 2C). When the ampulla potential had shifted to a more negative level with cessation of afferent discharge, the same cathodal stimulus produced an even smaller RP and almost no GP in the terminal (Fig. 2D). 
An antidromic spike superimposed on the depolarizing GP either failed to invade the terminal (Fig. 2A) or was diminished in height (B), suggesting a conductance increase which is expected for the excitatory PSP. ${ }^{9)}$ (The antidromic stimulation of the nerve trunk involves only the afferent nerve, because in this preparation the efferent or inhibitory innervation is absent, unlike in the ordinary lateralis receptor. ${ }^{8)}$ ) During the hyperpolarizing GP, however, the antidromic spike was never blocked, but even increased its peak height, suggesting a decreased conductance (Fig. 2C and D). This was supplemented in part by a direct conductance measurement with the bridge method. Namely the conductance of the afferent terminal never increased during the hyperpolarizing GP, while a marked increase was observed during the depolarizing GP. The hyperpolarizing GP thus can not be regarded as an inhibitory PSP, although the inactivating process for the spike initiation during the slow potential shift must be also taken into account.

Similar hyperpolarizing slow potentials which are not associated with the conductance increase have been reported to result from the electrogenic ionic pump mechanism in several instances. ${ }^{7)}$ However, this mechanism is not likely to be involved in the present case. The hyperpolarizing GP usually showed a distinct inflexion at its start (Fig. 2C), suggesting an abolition of a pre-existing process. There was almost no hyperpolarizing GP, when the potentials of the ampulla and hence of the afferent terminal had been shifted to a lower level (Fig. 2D). The amplitude of the GP was dependent directly on the $\mathrm{RP}$, but not affected by the presence or absence of the afferent discharge (Fig. 1, D to F).

The simplest explanation therefore would be that the hyperpolarizing GP is due to a disfacilitation, or repolarization from the previous level of steady depolarization which has been caused by a maintained effect of an excitatory transmitter. The abrupt start of the hyperpolarizing GP shown already further suggests that the maintained transmitter action be not due to an accumulation of the transmitter, but due to a steady release of which. With respect to the mechanism responsible for the maintained release of transmitter, experimental data are less conclusive at present and will be discussed more fully in another paper. However, there are indications that the maintained transmitter release is in turn caused by an asychronous and spontaneous activity of the electrically excitable membrane at the innervated face of receptor cells, rather than by the quantal release process from the quiescent presynaptic element. An example indicative of the receptor spontaneity is also shown in Fig. 2. The ampulla often shows a gradual fluctuation of a few $\mathrm{mV}$ either spontaneously 
or in response to stimulation. The RP to a constant current pulse during the fluctuation is nonlinear and dependent both on stimulus polarity and on pre-stimulus level of the ampulla potential. For the present purpose it will suffice to note the difference of the responses on cathodal stimulation at two levels. It is clearly larger at the control level (Fig. 2, C) than at the lowest or fully suppressed level (D), presumably because of abolition of the spontaneous depolarizing receptor activity at the control level. The suppression in terms of the afferent discharge frequency then can be attributed to the disfacilitation working at two levels, first at the receptor cell and then at the receptorafferent synapses.

Essentially similar mode of operation has been proposed for the isolated ampullae of Lorenzini of the skate, in which the hyperpolarizing GP was only occasionally observed, but the conductance measurement was not carried out.9) The electrical excitability, although unknown for the sensory receptors of other modalities, has been reported also in the phasic electroreceptors of teleost. ${ }^{1)}$

Acknowledgement. The authors are particularly grateful to the staff of Mito Aquarium, Numazu, and of Kanazawa Aquarium, Kanazawa, for the supply and maintenance of the material. A part of the work has been performed in Misaki Marine Biological Station, the director of which laboratory, Prof. T. Kobayashi is acknowledged for kindly providing us with the laboratoy space and material. The work was supported in part by grants from the Ministry of Education (Y.O.) 7004, 90958, 96050 and 99054, and from the NIH (Y.O.) NS-07201-03, and also by Teikyo University.

\section{References}

1) Bennett, M. V. L. (1965) : Cold Spring Harbor Symp. Quant. Biol., 30, 245.

2) Flock, A., and Wersäll, J. (1962) : J. Cell Biol., 15, 19.

3) Fouries, M. G. F. (1971): Handbook of Sensory Physiology, 1, 243. (Autrum, H., Jung, R., Loewenstein, W. R., Mac Kay, D. M., and Teuber, H. L., ed.) Springer-Verlag (see pp. 251-255).

4) Friedrich-Freska, H. (1930): Zool. Anz., 87, 49.

5) Furukawa, T., and Ishii, Y. (1967): J. Neurophysiol., 30, 1377.

6) Murray, R. W. (1965) : J. Physiol. (Lond.), 180, 592.

7) Nakajima, S., and Takahashi, K. (1969): J. Physiol. (Lond.), 187, 105.

8) Nishihara, H., and Yamamoto, Y. (1971): J. Electron Microscop. (English Abst.), 20, 231.

9) Obara, S., and Bennett, M. V. L. (1972): J. Gen. Physiol., 60, 534.

10) Obara, S., and Oomura, Y. (1972): J. Physiol. Soc. Japan (English Abst.), 34, 599 . 